\title{
Temporal persistence and long-term effect on noninvasive prenatal testing by residual fetal cell-free DNA of demised co-twin after selective fetal reduction in dichorionic diamniotic twin pregnancies
}

\author{
Min Chen ${ }^{1}$, Fengxia Su${ }^{2}$, Jia Wang ${ }^{1}$, Li Zhou ${ }^{3}$, Qiang $\mathrm{Liu}^{3}$, Xiang Chai ${ }^{3}, \mathrm{Yu} \mathrm{Yuan}^{3}$, Miao \\ $\mathrm{Cen}^{3}$, Yujing $\mathrm{Wu}^{2}$, Yi Wang ${ }^{4}$, Yan Zhang ${ }^{4}$, Fang $\mathrm{Chen}^{2}$, Dunjin $\mathrm{Chen}^{5}$, and $\mathrm{Ya} \mathrm{Gao}^{2}$ \\ ${ }^{1}$ Department of Fetal Medicine and Prenatal Diagnosis, The Third Affiliated Hospital of \\ Guangzhou Medical University \\ ${ }^{2}$ BGI-Shenzhen \\ ${ }^{3}$ Clinical Laboratory of BGI Health, BGI-Shenzhen \\ ${ }^{4}$ MGI, BGI-Shenzhen, \\ ${ }^{5}$ Department of Obstetrics, The Third Affiliated Hospital of Guangzhou Medical University
}

April 28, 2020

\begin{abstract}
Objectives: To determine the temporal persistence of residual cell-free DNA (cfDNA) of deceased co-twin in maternal circulation after selective fetal reduction and evaluate its long-lasting effect on noninvasive prenatal testing (NIPT) results. Design: Prospective observational study Setting: The Third Affiliated Hospital of Guangzhou Medical University Population: Dichorionic diamniotic twins $(\mathrm{n}=5)$ underwent selective fetal reduction of a co-twin with trisomy. Methods: With consent, maternal blood was collected immediately before reduction and periodically after reduction until birth. CfDNA of each maternal blood sample was sequenced for NIPT and analyzed for fetal trisomies and fetal fractions. Main Outcome Measured: Detectable T-scores for trisomy identification and three types of fetal fractions including the total fetal fraction, the fetal fraction of the deceased co-twin, and the fetal fraction of the surviving co-twin.
\end{abstract}

\section{Introduction}

The fetal demise of a co-twin (i.e., spontaneous reduction to a singleton pregnancy) is common with an estimated prevalence of $6.2 \%$ in all twin pregnancies $[1,2]$. This prevalence might be underestimated, since the fetal demise occurred in the early trimester, also known as the 'vanishing twin', which may affect as high as $30 \%$ of early diagnosed pregnancies [3-6]. The reasons for fetal demise could be complicated, yet chromosomal anomaly could be an important factor, given the high rate of chromosome aneuploidy in early singleton miscarriages [7-9]. It has been previously reported that the demised fetus continues to give rise residual cell-free DNA (cfDNA) to maternal circulation, which can cause false-positive results of fetal aneuploidy and fetal gender when noninvasive prenatal testing (NIPT) is conducted [10-12]. This poses technical uncertainties and counseling difficulties when providing NIPT service in twin pregnancies, or pregnancies with unaware fetus demise. So far, there is a lack of clinical guidelines in terms of the interval after fetus demise to provide NIPT, partly because the longitudinal change of residual cfDNA of demise fetus has not been extensively studied. We aimed to use dichorionic diamniotic (DCDA) twin pregnancies undergoing selective fetal reduction of an aneuploidy co-twin as a model of natural fetal demise to investigate the sequential changes of residual cfDNA of the deceased fetus, as well as the persistent effect on NIPT.

\section{Methods}




\section{Study population and study design}

This was a prospective observational study in women of DCDA twin pregnancy opted for selective reduction of a co-twin in the Department of Fetal Medicine and Prenatal Diagnosis, the Third Affiliated Hospital of Guangzhou Medical University, China. Each woman has been previously confirmed to carry dizygotic twin pregnancy by ultrasound, and opted for fetal reduction due to an aneuploidy co-twin diagnosed by chorionic villus sampling (CVS) or amniocentesis. Before reduction, each woman was given a detailed explanation of the study, and written informed consent of participating in this study was obtained. All women were above 18 years old, and their gestational ages were eight weeks or above at the time of joining the study. Chromosomal abnormalities have not been found in women and her husband, neither the couples have received a blood transfusion, organ transplantation, cell therapy, or immunotherapy before pregnancy. Fetal reductions were conducted by transabdominal intracardiac injection of potassium chloride as the standard of practice [13]. Immediately before and twice a month after reduction till birth, the maternal blood of each woman was collected for NIPT for fetal T21, T18, T13 to evaluate the residual cfDNA effect of the reduced fetus. The couple's age, gestational age at each time of blood sampling, ultrasound scanning results, prenatal diagnosis results, and other relevant clinical information were recorded. This study was approved by the Ethics Committee of the Third Affiliated Hospital of Guangzhou Medical University (no.2019096) and the Institute Review Board of BGI-Shenzhen (BGI-IRB 19081).

\section{Plasma cfDNA extraction and sequencing}

For each blood collection, five milliliters of peripheral blood was obtained from the participating woman in an ethylene diamine tetraacetic acid-anticoagulated (EDTA) tube. Plasma was separated within 8 hours following a two-step centrifugation protocol [14]. Briefly, blood was centrifugated at $1600 \mathrm{~g}$ for 10 minutes at $4^{\circ} \mathrm{C}$, and then the supernatant was transferred to a new Eppendorf tube for second centrifugation at 16000 $\mathrm{g}$ for 10 minutes at $4^{\circ} \mathrm{C}$. The supernatant was taken into a new tube and stored at $-80^{\circ} \mathrm{C}$ for future use. All subsequent procedures, including cfDNA isolation, library construction, and sequencing, were performed at an ISO/IEC 17025 certified laboratory of BGI-Shenzhen, China. CfDNA was extracted from 200uL plasma using a Micro DNA Kit (Tiangen) following the manufacturer's instructions. The extracted cfDNA was end-repaired and then ligated with adaptors for multiplex sequencing. The ligated products were subjected to 12 cycles of amplification using the Kapa HIFI hotstart ready master mix (Kapa Biosystems), followed by quantitation using the dsDNA HS Assay kit 2.0 (Invitrogen) by Qubit2.0 and Agilent High Sensitivity DNA Kit with a 2100 Bioanalyzer (Agilent Technologies). PCR products were then normalized and processed for circularization using MGIEasyTM DNA Library Prep Kit (MGI, China) following the manufacturer's instructions [15]. Briefly, PCR products were heat-denatured at $95{ }^{\circ} \mathrm{C}$ for 3 minutes to make single strand DNA circles (ssDNA circles), which were then mixed with reagents of MGIEasyTM DNA Library Prep Kit and incubated at $37 \operatorname{deg} \mathrm{C}$ for 30 minutes to complete the circularization. The resulting ssDNA circle was then used to generate DNA nanoballs (DNBs) by rolling circle amplification (RCA) [16]. After RCA and the formation of DNBs, the final product was measured by Qubit (Thermo Fisher) using the ssDNA HS Assay kit (Invitrogen), and loaded on a DNBSEQ-500 platform (MGI, China) for sequencing [17] following the manufacturer's instructions. Sequencing was conducted with a strategy of single-end 35 base pairs. The data that support the findings of this study have been deposited into CNSA (CNGB Nucleotide Sequence Archive) of CNGBdb with accession number CNP0000992 (https://db.cngb.org/cnsa/ ) upon the request of accession code.

\section{Fetal fraction calculation}

Three different methods to calculate fetal fractions were used in this study for different purposes, including an artificial neural network model named the FF-QuantSC [18], a Y-chromosome method [19], and the relative coverage of the trisomy chromosome. FF-QuantSC was used to calculate the overall fetal fraction of both co-twins. In pregnancies with one male co-twin, the Y-chromosome method was used to calculate the fetal fraction of the male fetus. In pregnancies with two male fetuses, the Y-chromosome method calculated the fetal fraction equal to an overall fetal fraction, and results were compared with the FF-QuantSC method. The relative coverage of the trisomy chromosome was specifically used to calculate the fetal fraction of the 
trisomy co-twin who was terminated by the selective reduction.

FF-QuantSC exploits an artificial neural network to calculate the fetal fraction of both male and female cotwins [18]. Briefly, a fully connected neural network model with a single hidden layer and 128 neurons was trained by the Vapnik-Chervonenkis dimension [20] and trained with over 100 thousand of male pregnancies. Sequencing reads were partitioned into continuous genomic windows of $60 \mathrm{~kb}$ in length for feature selection. Standardization by within-sample z-score transformation was then used to generate a final feature matrix. After training, the FF-QuantSC model was testified in six testing groups of about 240 thousand pregnancies, including 36 thousand twin pregnancies and 80 thousand female pregnancies [18].

Y-chromosome method calculated fetal fractions using unique sequence reads from the Y chromosome (eq.1).

Fetal fraction $=2 * \frac{\mathrm{UR}_{Y}}{\mathrm{UR}}(1)$

where $\mathrm{UR}_{Y}$ represents the unique reads on chromosome $\mathrm{Y}$, and URrepresents the unique reads on autosome chromosomes.

In pregnancies with a trisomy co-twin, fetal fraction was calculated using the sequencing reads of the trisomic chromosome, typically chromosome 13, 18, or 21 (eq.2).

$\varepsilon_{i}=2 \times \frac{\mathrm{UR}_{i}-\mathrm{UR}}{\mathrm{UR}} i=$ chromosome 13,18 , or $21(2)$

Where $\varepsilon_{i, Y}$ represents the fetal fraction estimate by chromosome $i$; $U R_{i}$ represents the unique reads of chromosome $i$; UR represents the mean of unique reads on all autosomal chromosomes.

\section{Chromosome trisomy risk calculation}

Sequencing data were analyzed using a binary hypothesis T-score as previously described [19]. Briefly, a binary hypothesis T-test and logarithmic likelihood ratio L-score between the two t-tests were used to classify whether the fetuses had T21, T18 or T13. T-score higher than 3 was used as the cut-off to identify fetal trisomy, and L-score higher than 1 was used to determine the confidence.

\section{Results}

\section{Patients}

From January 2017 to January 2018, five women carrying DCDA twins with one aneuploidy fetus were enrolled in this study (Table 1 ). The median maternal age was 38.8 years and $80 \%(4 / 5)$ of pregnant women had a maternal age above 35 years. All women were conceived by in-vitro fertilization and embryo transfer (IVF-ET), and each woman had received the transplant of two embryos. During routine prenatal healthcare, all women had at least one risk factor of fetal aneuploidy, including advanced maternal age, increased fetal nuchal translucency (NT) thickness, and structural abnormalities in ultrasound examination (Table 1). Fetal aneuploidies were confirmed by CVS or amniocentesis, showing four women with a co-twin of trisomy 18 (T18) and one woman with a co-twin of trisomy 21 (T21). The median of gestational age receiving reduction procedure was 15 weeks. After reduction, each woman voluntarily donated her blood samples every two to five weeks until delivery. Depending on their willingness, patients 1, 2, and 5 gave blood for seven times, five times, and six times after reduction, respectively. Patient 3 and 4 both gave blood for nine times after reduction.

\section{Fetal fractions}

The temporal dynamic changes of fetal fractions by three different methods in all five pregnancies are shown in Figure 1, and detailed information at each timepoints listed in Table S1.

In patient 1, both fetuses were female and fetal fractiosn could not be calculated using Y-chromosome signals. However, the fetal fraction of the reduced T18 fetus was calculated using trisomy signals, showing an immediate decline from $3.27 \%$ to $2.01 \%$ after reduction and a mild increase to $3.41 \%$ at eight weeks after 
reduction. After that, the demised T18 fetus gradually stopped the release of cfDNA at 16 weeks after reduction as the fetal fraction dropped to close to 0 . In contrast, after a transient decline, the overall fetal fraction maintained stable for several weeks, and then rise when the terminated fetus reduced the release of cfDNA at eight weeks after reduction.

In patient 2, who had a male euploidy and a male T18 fetus, the fetal fraction of the terminated T18 fetus increased from $1.80 \%$ to the maximum of $11.71 \%$ at nine weeks after reduction and then rapidly declined to close to 0 at 11 weeks after reduction. Since both fetuses were male, the male fetal fractions calculated by the Y-chromosome method and the overall fetal fractions calculated by FF-QuantSC were almost overlapped, which showed peak levels at nine weeks after reduction following the quick decline afterward. At 12 weeks after reduction when the terminated fetus stopped the release of cfDNA, the fetal fraction of the euploid fetus quickly rebound, and kept rising until birth. It was noted that at 15 and 20 weeks after reduction, the overall fetal fractions were slightly higher than the fetal fractions of male fetuses.

Both patient 3 and 4 had discordant sex twins with a female trisomy and a male euploid fetus. The fetal fractions of the terminated female fetus showed the increase of fetal fraction to peak levels at seven to nine weeks after reduction. After that, the terminated fetus in patient 3 gradually reduced the release of cfDNA and stopped discharge at 22 weeks after reduction, while the fetal fraction of the terminated fetus in patient 4 dropped to close 0 at 15 weeks after reduction. In both patients, once the fetal fractions of the terminated female fetuses began to decline, the fetal fractions of the euploid male fetuses rapidly increased, especially when the fetal fraction of the terminated female fetus was down to nearly 0 . When the terminated female fetuses in both patient 3 and 4 still gave rise substantial amount of cfDNA to maternal circulation, the overall fetal fractions were higher than the fetal fractions of each co-twin. However, after the fetal fractions of the terminated female fetuses reduced to a relatively low level, the total fetal fractions were closely correlated with the fetal fractions of the euploid male fetuses.

In patient 5, since a male T18 fetus was terminated in the female-male twins, the fetal fractions calculated with trisomy signals and Y chromosome were almost identical, both showing a transient decrease shortly after reduction and later rise to maximum levels at eight weeks after reduction, and finally decline to nearly $0 \%$ at 14 weeks after reduction. Before seven weeks of post-reduction, the overall fetal fraction maintained two to four folds higher than the fetal fraction of the terminated fetus. Once the terminated fetus stopped the release of cfDNA at 14 weeks after reduction, the overall fetal fraction began to increase rapidly.

\section{NIPT results}

As a result of the persistence of a high fetal fraction of demised trisomy co-twin, NIPT T-scores of the remaining euploid fetuses in all five pregnancies were compromised for long periods after reduction (Figure 2a). Detailed NIPT results of each time points are listed in Table S1.

Patient 1 had her T-score of chromosome 18 reduced from 7.27 before reduction to 4.55 at three weeks after reduction, and later rise to 8.20 at eight weeks after reduction. Then her T-score declined to 1.24 at 16 weeks after reduction and her NIPT results remained T18 low-risk afterward.

Patient 2 had her T-score of chromosome 18 dramatically rise from 4.55 at two weeks after reduction to 24.62 at nine weeks after reduction, and then rapidly declined to 2.09 at 12 weeks after reduction for T18 low-risk afterward. Interestingly, her T-score of chromosome 13 reached 3.33 at 15 weeks after reduction, and then rise to 5.65 at 20 weeks after reduction, indicating high risk of trisomy 13 (T13) (Figure 2b). This was consistent with the results of her fetal fraction, showing that at 15 and 20 weeks post-reduction, the overall fetal fraction calculated by FF-QuantSC was slightly higher than the fetal fractions of both male fetuses calculated by the Y-chromosome method. A later confirmation in placenta tissues by sequencing placental tissues validated the presence of placenta mosaicism of T13 (Figure 2c).

In patient 3 , in the first two weeks after reduction, her T-score of chromosome 21 only mildly increased from 10.04 to 13.94. After that, her T-score reached a maximum of 36.60 at eight weeks after reduction, and then quickly declined to 8.76 at 12 weeks after reduction. Since then, her NIPT results maintained high risk for 
T21 for a very long time until 22 weeks after reduction.

In patient 4, her T-score of chromosome 18 also showed an initial mild but later faster increase, and reached a maximum of 17.27 at seven weeks after reduction. After that, the T-score gradually declined to 1.85 at 15 weeks after reduction. Since then, the NIPT results changed to T18 low risk.

Patient 5 had her T-score of chromosome 18 reduced from 12.39 before reduction to 4.84 at two weeks after reduction, and rise to $16.04 \%$ at seven weeks after reduction. The T-score then continuously declined, and the NIPT results changed to T18 low-risk at 14 weeks after reduction.

\section{Prognosis}

All five women gave live birth to a healthy baby at 37 to 39 gestational weeks without neonatal abnormalities.

\section{Discussion}

\section{Main findings}

Using selective reduction of a co-twin with aneuploidy as the simulation of twin demise, we demonstrated that the fetal fraction of the terminated fetus underwent dynamic changes and persistent effect throughout pregnancy. After fetal reduction, the fetal fraction of the terminated fetus took 7 to 9 weeks to reach a peak level, and then gradually dropped down to nearly $0 \%$ at 15 to 16 weeks after reduction. In one case (patient 3 ), the residual cfDNA of the terminated fetus even gave rise to observable fetal fraction at 20 weeks after reduction, equivalent to 37 gestational weeks in this woman. On the other hand, in patient 3 and 4 , we observed that the fetal fraction of the remaining normal fetus appeared to maintain stable or mildly increase until the fetal fraction of the trisomy co-twin declined.

The influence of the residual cfDNA of a terminated fetus was apparent, as we found the persistent detection of fetal trisomy after the fetal reduction, ranging from 12 to 22 weeks post-reduction with the median of 15.8 weeks. Importantly, in all pregnancies, T-scores demonstrated close correlations to the fetal fractions of the terminated fetus, showing a rapid increase from two to three weeks after reduction and reached the maximum values at seven to nine weeks after reduction, following the gradual decline to close to 0 in the late third trimester. In patient 3 who had a very long presence of residual cfDNA of the terminated T21 fetus, NIPT showed positive results until 20 weeks after reduction, which was concordant to the fetal fraction results.

\section{Strengths and limitations}

The major strength of this study was the consecutive observational approach and prospective collection of blood samples. Previously, several studies have reported the disturbance of NIPT by residual cfDNA of twin demise $[11,21,22]$. However, very few studies demonstrate the longitudinal effect of residual cfDNA after twin demise. We successfully established the consecutive profiles of residual cfDNA changes after twin demise by densely testing blood samples until birth, which provides the robust evidence to elucidate an important question: how long the placenta of a deceased co-twin can release cfDNA into the maternal circulation? Another strength of this study was a combination of three different methods of calculating fetal fractions. This helps compare the total fetal fraction measured by FF-QuantSC, the fetal fraction of the demised cotwin measured by relative coverage of the trisomy chromosome, and in two cases (patient 3 and 4) the fetal fraction of the survived euploid fetuses measured by Y-chromosome.

The limitations of this study included a small sample size and the restriction of selecting DCDA twins with a trisomy co-twin to simulate a natural twin demise. Due to the small sample size, statistical analysis could not be conducted. For the ease of analyzing the individual fetal fraction of different co-twins, we only used dizygotic twins in our study. It is not clear if the same observations can be replicated in monozygotic twins, especially monochorionic twins who have a significantly higher risk of fetal demise [23, 24]. Moreover, the results obtained using the reduced trisomy co-twin might not fully represent natural twin demise, which can be caused by many other reasons with or without chromosomal abnormalities [7, 25]. Besides, in our study, women opted for reduction at 14 to 17 gestational weeks, thus missing the opportunities to observe residual 
cfDNA at early pregnancy when most vanishing twins occur. Lastly, because of the difficulties during clinical practice, the time points of sample collection in each woman were not identical. In one case (patient 2), the blood sample before the reduction was missing.

\section{Interpretations}

At the first two to three weeks after fetal reduction, there were discordant observations of fetal fraction changes, as two cases showed a bland increment of cfDNA release of demised co-twin while two other cases showed a slight decline. After that, all cases demonstrated consistent patterns of fast increase of a fetal fraction of demised co-twin, and reached the top level at 7-9 weeks after reduction and the cfDNA disappeared at 12-16 weeks after a demise or even longer. This indicates that the fetal demise induced increased release of residual cfDNA into maternal circulation for a substantial period. A recent study by Bevilacqua et al. may observe the a similar phenomenon [26], in which the fetal aneuploidy risk of NIPT after the selective reduction of a trisomy co-twin also displayed the 'rise-and-drop' changes in two cases.

Recently, Boyd described the histopathological changes of the placenta in intrauterine demise [27]. After fetal demise, maternal perfusion and fetal blood flow pressure disappear, which leads to the compromise of villous capillary integrity and endothelial karyorrhexis, eventually resulting in fibrosis avascular villi and solid fabrin deposition of intervillous space. Meanwhile, villous edema and intravillous hemorrhage accompanied by maternal inflammation could also occur to increase organ autolysis [27]. It was believed that intravascular karyorrhexis and multifocal villous obliteration might occur soon after fetal demise, whereas extensive obliteration of stem villi and have the level of villous fibrosis may take 14 days or more [27, 28]. Thus, we speculated that the initial discordant change patterns of residual cfDNA released from demised co-twin reflects the complicity of placental autolysis immediately after the cease of fetal blood flow and maternal perfusion. In contrast, the afterward rapid increase of residual cfDNA release was the result of extensive villous fibrosis and stem villi obliteration occurred at least 14 days after fetal demise. Also, it appeared that the fetal fraction of the surviving euploid fetus increased a lot faster when the release of residual cfDNA from the demised fetus declined at 7-9 weeks after reduction. This may reflect that the normal development and cfDNA emitting of the surviving fetus is influenced by the demised fetus.

Gestational age at fetal demise might have important roles in determining how long the residual cfDNA of the demised fetus can last. Gromminger et al. reported a vanishing twin that cfDNA of the demised co-twin only contributed to a small proportion of total fetal fraction, presumably because of early pregnancy demise [22]. However, another case reported by Niles et al. demonstrated different findings, in which a co-twin demised at $6+1$ weeks showed persistent false positive NIPT results for at least 15 weeks after fetal demise [29]. Our study added no evidence since the lack of samples with fetal demise at early pregnancy. Hence, further studies are still needed to explain the effect of gestational age on residual cfDNA clearance.

The fetal demise of a co-twin has been known as an important factor causing false positive NIPT results $[30,31]$. It has been estimated that a substantial proportion of false positive NIPT results ranging from $15 \%$ to $33 \%$ might be caused by the fetal demise of unaware twin pregnancies [32-34]. A key concern for clinical practice of providing NIPT service is how long the residual cfDNA from the demise co-twin remains in maternal circulation, and whether NIPT should be provided if the fetal demise of a co-twin is identified. Two previous studies have provided preliminary evidence. Curnow et al. demonstrated in five vanishing twins that the fetal cfDNA from vanished twins was detectable at eight weeks after demise [21]. However, their study was based on retrospective NIPT data of samples known with a fetus deceased at seven to eight weeks, and thus provided no follow-up of the rest of pregnancy. Bevilacqua et al. also used fetal reduction of a trisomic co-twin as a model for natural demise, and reported the prospective cfDNA testing results in seven cases [26]. However, due to minimal sampling frequency, although they found transiently detectable aneuploidy signals at three to eight weeks following reduction, they could not conclude the predictable patterns of aneuploidy risk and fetal fraction. In contrast, our study demonstrated that after fetal reduction, there was a consistent pattern of the change of fetal trisomy T-score and fetal fraction.

Both the fetal fraction of demised fetus and the trisomy T-score increased to a peak level at seven to nine 
weeks after reduction, possibly because of the increased rate of placental tissue autolysis after fetus deceased. Although continuous decline after eight weeks post-reduction, the detectable fetal fraction of demised fetus and trisomy T-score still lasted from 12 to 16 weeks after reduction, and even up to 20 weeks post-reduction. This finding is consistent with a sporadic case showing NIPT false positive results of fetal sex and aneuploidy at 15 weeks after a demised co-twin [29]. However, this phenomenon has not been thoroughly proven. Based on our results, residual cfDNA of fetal demise remained in maternal circulation had a long-lasting effect on NIPT by inducing false positive results for a long time of pregnancy. Therefore, the current data does not support providing NIPT to pregnancies given the identification of fetal demise.

\section{Conclusions}

Continuous detection of a significant risk of fetal trisomy after fetal reduction indicated that residual cfDNA remained in maternal plasma for around 12 to 22 weeks after fetal demise. The persistent detection of residual cfDNA of the demised co-twin has a long-lasting influence on the results of NIPT. It should be cautious about providing NIPT in pregnancies with a risk of fetal demise.

\section{Conflicts of Interest}

None of the authors have any conflicts of interest to declare. Completed disclosure of interest forms is available to view online as supporting information.

\section{Contribution to authorship}

MC and YG designed the study. FS and JW wrote the first version of the manuscript. MC and JW recruited patents and collected samples and clinical follow-up. FS, YW, YW, YZ, FC performed sample preparation and sequencing experiments. LZ, QL, XC, YY, MC conducted bioinformatics analysis. MC, FS, JW, and YG interpreted sequencing results and clinical implications. MC, DC and YG overviewed the study, finalized the manuscript, and provided funding.

\section{Details of ethics approval}

This study was approved on May 21, 2019 by the Ethics Committee of the Third Affiliated Hospital of Guangzhou Medical University (approval no.2019096) and the Institute Review Board of BGI-Shenzhen (approval no.19081).

\section{Funding}

The study was supported by the National Key Research and Development Program of China (2018YFC 1004104), the National Natural Science Foundation of China (NSFC) (No. 81671470), and Shenzhen Municipal Government of China (JCYJ20180703093402288 and JCYJ20170412153136375).

\section{Acknowledgments}

We thank all the participating women for their cooperation in this study. We thank Dr. Tze Kin Lau for valuable suggestions in revising the manuscript.

\section{Suppporting Information}

Additional supporting information may be found online in the Supporting Information section at the end of the article.

Table S1. Detailed information of patients that had NIPT-positive results.

\section{Reference}

1. Ong SS, Zamora J, Khan KS, Kilby MD: Prognosis for the co-twin following single-twin death: a systematic review. BJOG2006, 113 (9):992-998.

2. Mackie FL, Rigby A, Morris RK, Kilby MD: Prognosis of the co-twin following spontaneous single intrauterine fetal death in twin pregnancies: a systematic review and meta-analysis . BJOG 
2019,126 (5):569-578.

3. Sampson A, de Crespigny LC: Vanishing twins: the frequency of spontaneous fetal reduction of a twin pregnancy . Ultrasound Obstet Gynecol 1992, 2 (2):107-109.

4. Landy HJ, Weiner S, Corson SL, Batzer FR, Bolognese RJ: The "vanishing twin": ultrasonographic assessment of fetal disappearance in the first trimester. Am J Obstet Gynecol 1986,155 (1):14-19.

5. Kelly MP, Molo MW, Maclin VM, Binor Z, Rawlins RG, Radwanska E:Human chorionic gonadotropin rise in normal and vanishing twin pregnancies . Fertil Steril 1991, 56 (2):221-224.

6. Dickey RP, Taylor SN, Lu PY, Sartor BM, Storment JM, Rye PH, Pelletier WD, Zender JL, Matulich EM: Spontaneous reduction of multiple pregnancy: incidence and effect on outcome . $A m J$ Obstet Gynecol 2002, 186 (1):77-83.

7. Shek NW, Hillman SC, Kilby MD: Single-twin demise: pregnancy outcome . Best practice $\mathcal{E}^{3}$ research Clinical obstetrics \& gynaecology 2014, 28 (2):249-263.

8. Hansen M, Kurinczuk JJ, Milne E, de Klerk N, Bower C:Assisted reproductive technology and birth defects: a systematic review and meta-analysis . Hum Reprod Update 2013,19 (4):330-353.

9. Yukobowich E, Anteby EY, Cohen SM, Lavy Y, Granat M, Yagel S:Risk of fetal loss in twin pregnancies undergoing second trimester amniocentesis(1) . Obstet Gynecol 2001,98 (2):231-234.

10. Vlkova B, Hodosy J: Vanishing twin as a potential source of bias in non-invasive fetal sex determination: a case report . J Obstet Gynaecol Res 2014, 40 (4):1128-1131.

11. Hochstenbach R, Elferink MG, van Zon PHA, Lichtenbelt KD, van Harssel J, Schuring-Blom H, PageChristiaens G: Discordant NIPT result in a viable trisomy-21 pregnancy due to prolonged contribution to cfDNA by a demised trisomy-14 cotwin . Clin Case Rep 2018,6 (5):788-791.

12. Kelley JF, Henning G, Ambrose A, Adelman A: Vanished Twins and Misdiagnosed Sex: A Case Report with Implications in Prenatal Counseling Using Noninvasive Cell-Free DNA Screening . J Am Board Fam Med 2016, 29 (3):411-413.

13. Khalil A, Rodgers M, Baschat A, Bhide A, Gratacos E, Hecher K, Kilby MD, Lewi L, Nicolaides KH, Oepkes D et al : ISUOG Practice Guidelines: role of ultrasound in twin pregnancy . Ultrasound Obstet Gynecol 2016, 47 (2):247-263.

14. Lau TK, Cheung SW, Lo PS, Pursley AN, Chan MK, Jiang F, Zhang H, Wang W, Jong LF, Yuen OK et al : Non-invasive prenatal testing for fetal chromosomal abnormalities by low-coverage whole-genome sequencing of maternal plasma DNA: review of 1982 consecutive cases in a single center. Ultrasound Obstet Gynecol 2014,43 (3):254-264.

15. Xu Y, Lin Z, Tang C, Tang Y, Cai Y, Zhong H, Wang X, Zhang W, Xu C, Wang J et al : A new massively parallel nanoball sequencing platform for whole exome research . BMC bioinformatics 2019 , 20 (1):153.

16. Drmanac R, Sparks AB, Callow MJ, Halpern AL, Burns NL, Kermani BG, Carnevali P, Nazarenko I, Nilsen GB, Yeung G et al : Human genome sequencing using unchained base reads on selfassembling DNA nanoarrays . Science 2010, 327 (5961):78-81.

17. Huang J, Liang X, Xuan Y, Geng C, Li Y, Lu H, Qu S, Mei X, Chen H, Yu T et al : A reference human genome dataset of the BGISEQ-500 sequencer. GigaScience 2017, 6 (5):1-9.

18. Yuan Y, Chai X, Liu N, Gu B, Li S, Gao Y, Zhou L, Liu Q, Yang F, Liu J et al : FF-QuantSC: accurate quantification of fetal fraction by a neural network model . Mol Genet Genomic Med2020:e1232. 
19. Jiang F, Ren J, Chen F, Zhou Y, Xie J, Dan S, Su Y, Yin B, Su W, Zhang H et al : Noninvasive Fetal Trisomy (NIFTY) test: an advanced noninvasive prenatal diagnosis methodology for fetal autosomal and sex chromosomal aneuploidies . BMC Med Genomics 2012,5 :57.

20. Cherkassky V: The nature of statistical learning theory ${ }^{\sim}$. IEEE Trans Neural Netw 1997,8 (6):1564.

21. Curnow KJ, Wilkins-Haug L, Ryan A, Kirkizlar E, Stosic M, Hall MP, Sigurjonsson S, Demko Z, Rabinowitz M, Gross SJ: Detection of triploid, molar, and vanishing twin pregnancies by a singlenucleotide polymorphism-based noninvasive prenatal test . Am J Obstet Gynecol 2015, 212 (1):79 e71-79.

22. Gromminger S, Yagmur E, Erkan S, Nagy S, Schock U, Bonnet J, Smerdka P, Ehrich M, Wegner RD, Hofmann W et al : Fetal Aneuploidy Detection by Cell-Free DNA Sequencing for Multiple Pregnancies and Quality Issues with Vanishing Twins . J Clin Med 2014,3 (3):679-692.

23. Kaufman HK, Hume RF, Jr., Calhoun BC, Carlson N, Yorke V, Elliott D, Evans MI: Natural history of twin gestation complicated by in utero fetal demise: associations of chorionicity, prematurity, and maternal morbidity. Fetal Diagn Ther 2003,18 (6):442-446.

24. McPherson JA, Odibo AO, Shanks AL, Roehl KA, Macones GA, Cahill AG:Impact of chorionicity on risk and timing of intrauterine fetal demise in twin pregnancies . Am J Obstet Gynecol 2012,207 (3):190 e191-196.

25. Kilby MD, Govind A, O'Brien PM: Outcome of twin pregnancies complicated by a single intrauterine death: a comparison with viable twin pregnancies . Obstet Gynecol 1994, 84 (1):107109.

26. Bevilacqua E, Chen K, Wang Y, Doshi J, White K, de Marchin J, Conotte S, Jani JC, Schmid M: Cell-free DNA analysis after reduction in multifetal pregnancy . Ultrasound Obstet Gynecol2020, 55 (1):132-133.

27. Boyd TK: The placenta in intrauterine demise . APMIS2018, 126 (7):621-625.

28. Genest DR: Estimating the time of death in stillborn fetuses: II. Histologic evaluation of the placenta; a study of $\mathbf{7 1}$ stillborns . Obstet Gynecol 1992, 80 (4):585-592.

29. Niles KM, Murji A, Chitayat D: Prolonged duration of persistent cell-free fetal DNA from vanishing twin. Ultrasound Obstet Gynecol 2018, 52 (4):547-548.

30. Wilkins-Haug L, Zhang C, Cerveira E, Ryan M, Mil-Homens A, Zhu Q, Reddi H, Lee C, Bianchi DW: Biological explanations for discordant noninvasive prenatal test results: Preliminary data and lessons learned . Prenat Diagn 2018, 38 (6):445-458.

31. Cuckle H, Benn P, Pergament E: Cell-free DNA screening for fetal aneuploidy as a clinical service . Clin Biochem 2015,48 (15):932-941.

32. Futch T, Spinosa J, Bhatt S, de Feo E, Rava RP, Sehnert AJ:Initial clinical laboratory experience in noninvasive prenatal testing for fetal aneuploidy from maternal plasma DNA samples .Prenat Diagn 2013, 33 (6):569-574.

33. Porreco RP, Garite TJ, Maurel K, Marusiak B, Obstetrix Collaborative Research N, Ehrich M, van den Boom D, Deciu C, Bombard A:Noninvasive prenatal screening for fetal trisomies 21, 18, 13 and the common sex chromosome aneuploidies from maternal blood using massively parallel genomic sequencing of DNA . Am J Obstet Gynecol 2014, 211 (4):365 e361-312.

34. Hartwig TS, Ambye L, Sorensen S, Jorgensen FS: Discordant non-invasive prenatal testing (NIPT) - a systematic review .Prenat Diagn 2017, 37 (6):527-539. 
Table1 Characteristics and clinical details of pregnancies. ART, artificial reproductive technology; DCDA, dichorionic diamniotic; IVF-ET, in vitro fertilization and embryo transfer; NT, nuchal translucency.

\begin{tabular}{|c|c|c|c|c|c|}
\hline Samples & Patient 1 & Patient 2 & Patient 3 & Patient 4 & Patient 5 \\
\hline $\begin{array}{l}\text { Maternal } \\
\text { age (year) }\end{array}$ & 42 & 40 & 31 & 44 & 37 \\
\hline $\begin{array}{l}\text { Pregnancy } \\
\text { history }\end{array}$ & G1P0 & G3P0 & G1P0 & $\mathrm{G} 2 \mathrm{P} 1$ & $\mathrm{G} 2 \mathrm{P} 1$ \\
\hline Chorionicity & DCDA & DCDA & DCDA & DCDA & DCDA \\
\hline $\begin{array}{l}\text { ART } \\
\text { method }\end{array}$ & IVF-ET & IVF-ET & IVF-ET & IVF-ET & IVF-ET \\
\hline $\begin{array}{l}\text { Number of } \\
\text { embryo } \\
\text { transfer }\end{array}$ & 2 & 2 & 2 & 2 & 2 \\
\hline $\begin{array}{l}\text { Gestational } \\
\text { age for } \\
\text { ultrasound } \\
\text { (week) }\end{array}$ & 13 & 14 & $12+5$ & $12+6$ & $12+5$ \\
\hline $\begin{array}{l}\text { Ultrasound } \\
\text { examination }\end{array}$ & $\begin{array}{l}\text { Fetus 1: NT } \\
\text { 1.5mm; Fetus } 2: \\
\text { NT } 4.3 \mathrm{~mm} \text {, } \\
\text { nasal bone loss, } \\
\text { tricuspid } \\
\text { regurgitation, } \\
\text { single umbilical } \\
\text { artery }\end{array}$ & $\begin{array}{l}\text { Fetus 1: NT } \\
2.0 \mathrm{~mm} \text {; Fetus } 2 \text { : } \\
\text { NT } 3.5 \mathrm{~mm} \text {, } \\
\text { nasal bone loss, } \\
\text { omphalocele }\end{array}$ & $\begin{array}{l}\text { Fetus 1: NT } \\
1.8 \mathrm{~mm} \text {; Fetus } 2 \text { : } \\
\text { NT } 2.0 \mathrm{~mm} \text {, } \\
\text { nasal bone loss }\end{array}$ & $\begin{array}{l}\text { Fetus 1: NT } \\
2.7 \mathrm{~mm} \text {, nasal } \\
\text { bone loss, } \\
\text { tricuspid } \\
\text { regurgitation, } \\
\text { single umbilical } \\
\text { artery; Fetus 2: } \\
\text { NT 2.5mm }\end{array}$ & $\begin{array}{l}\text { Fetus 1: NT } \\
3.3 \mathrm{~mm} \text {; Fetus 2: } \\
\text { NT 2.6mm, } \\
\text { nasal bone loss, } \\
\text { omphalocele }\end{array}$ \\
\hline $\begin{array}{l}\text { Prenatal } \\
\text { diagnosis }\end{array}$ & $\begin{array}{l}\text { Fetus 1: } 46, \mathrm{XX} ; \\
\text { Fetus 2: } 47, \mathrm{XX} \text {, } \\
+18\end{array}$ & $\begin{array}{l}\text { Fetus 1: } 46, \mathrm{XY} \\
\text { Fetus 2: } 47, \mathrm{XY} \\
+18\end{array}$ & $\begin{array}{l}\text { Fetus 1: } 46, \mathrm{XY} ; \\
\text { Fetus 2: } 47, \mathrm{XX} \\
+21\end{array}$ & $\begin{array}{l}\text { Fetus 1: } 47, \mathrm{XX} \\
+18 ; \text { Fetus } 2: \\
46, \mathrm{XY}\end{array}$ & $\begin{array}{l}\text { Fetus 1: } 46, \mathrm{XX} \text {; } \\
\text { Fetus 2: } 47, \mathrm{XY} \text {, } \\
+18\end{array}$ \\
\hline $\begin{array}{l}\text { Gestational } \\
\text { age for } \\
\text { reduction } \\
\text { surgery } \\
\text { (week) }\end{array}$ & $14+3$ & $14+0$ & $17+4$ & $15+5$ & $14+5$ \\
\hline Delivery & $\begin{array}{l}\text { One female } \\
\text { with no } \\
\text { apparent } \\
\text { abnormalities }\end{array}$ & $\begin{array}{l}\text { One male with } \\
\text { no apparent } \\
\text { abnormalities }\end{array}$ & $\begin{array}{l}\text { One male with } \\
\text { no apparent } \\
\text { abnormalities }\end{array}$ & $\begin{array}{l}\text { One male with } \\
\text { no apparent } \\
\text { abnormalities }\end{array}$ & $\begin{array}{l}\text { One female } \\
\text { with no } \\
\text { apparent } \\
\text { abnormalities }\end{array}$ \\
\hline $\begin{array}{l}\text { Gestational } \\
\text { age of birth } \\
\text { (weeks) }\end{array}$ & $38+6$ & $36+5$ & $39+2$ & $37+1$ & $38+5$ \\
\hline
\end{tabular}

\section{Hosted file}

Figure1. Fetal fractions at multiple time points before and after fetal reduction in five twin pregnanc available at https://authorea.com/users/316246/articles/446393-temporal-persistence-and-longterm-effect-on-noninvasive-prenatal-testing-by-residual-fetal-cell-free-dna-of-demised-cotwin-after-selective-fetal-reduction-in-dichorionic-diamniotic-twin-pregnancies

\section{Hosted file}

Figure2.NIPT results by T-scores at each timepoint in five twin pregnancies.docx available at 
https://authorea.com/users/316246/articles/446393-temporal-persistence-and-long-term-effecton-noninvasive-prenatal-testing-by-residual-fetal-cell-free-dna-of-demised-co-twin-afterselective-fetal-reduction-in-dichorionic-diamniotic-twin-pregnancies 